\title{
In vitro and in vivo drug release and antibacterial properties of the novel vancomycin-loaded bone-like hydroxyapatite/poly amino acid scaffold
}

This article was published in the following Dove Press journal:

International Journal of Nanomedicine

8 March 2017

Number of times this article has been viewed

\author{
Zhidong Cao' \\ Dianming Jiang ${ }^{2}$ \\ Ling $\mathrm{Yan}^{3}$ \\ Jun $\mathrm{Wu}^{4}$
}

'Department of Orthopedics, The Emergency Medical Center of Chongqing City, Chongqing, People's Republic of China; ${ }^{2}$ Department of Orthopedics, The First Affiliated Hospital of Chongqing Medical University, Chongqing, People's Republic of China; ${ }^{3}$ Department of Orthopedics, The First People's Hospital of Zunyi City, Zunyi, People's Republic of China; ${ }^{4}$ Department of Orthopedics, Children's Hospital Affiliated to Chongqing University of Medical Sciences, Chongqing, People's Republic of China
Correspondence: Dianming Jiang Department of Orthopedics, The First Affiliated Hospital of Chongqing Medical University, No I Youyi Road Yuzhong District, Chongqing City, People's Republic of China

$\mathrm{Tel}+86$ I388389430I

Email czd2445@sina.com
Abstract: Antibiotic-loaded carriers were developed to fill cavities and locally deliver antibiotics following implantation. However, the most commonly used antibiotic carrier, polymethyl methacrylate (PMMA), has many disadvantages including that it does not promote bone regeneration or conduction. Vancomycin-loaded bone-like hydroxyapatite/poly amino acid (V-BHA/PAA) was successfully fabricated by a homogeneous method, certified as biosafe and known to promote osteogenesis. To evaluate its drug-release features, the quantity of the vancomycin in the elution was obtained every 2 days after in vitro simulated body fluid immersion. The drug concentration in the elution was determined to obtain the drug-release curve. The in vitro drug release was a three-phase process with two release peaks. Its antibacterial activity was evaluated in vitro using an antibacterial zone assay, antibacterial inhibition, and scanning electron microscopy (SEM) observation. Scaffolds of V-BHA/PAA were implanted into a rabbit model of chronic osteomyelitis. The antibacterial activity of the material was evaluated in vivo by gross observations, X-ray, and histological and ultrastructural observations. During the first $48 \mathrm{~h}$, the vancomycin release was more rapid, followed by a period of sustained slow release. Use of V-BHA/PAA could achieve relatively long-term vancomycin delivery of 38 days in vitro and 42 days in vivo. V-BHA/PAA showed a significant and consistent bactericidal effect toward both Staphylococcus aureus and methicillin-resistant $S$. aureus (MRSA) in vitro and in vivo. Moreover, the bactericidal effect was stronger than that of vancomycin-loaded polymethyl methacrylate (V-PMMA). The duration of the antibacterial effect of V-BHA/PAA toward both $S$. aureus and MRSA exceeded 28 days in vitro, while that of V-PMMA lasted only 14 days. The curative rate for V-BHA/PAA in the chronic osteomyelitis model was $75 \%$ for regular $S$. aureus and $66.67 \%$ for MRSA infection, which significantly exceeded that of V-PMMA ( $50 \%$ and $41.67 \%$, respectively). Vancomycin released from the V-BHA/PAA scaffold was significantly superior to that delivered by V-PMMA.

Keywords: chronic osteomyelitis, vancomycin, drug release, scaffold, local antibiotics delivery, BHA/PAA

\section{Introduction}

Treatment of bone infections, especially chronic osteomyelitis, continues to trouble orthopedic surgeons not only because of the increased antibiotic drug resistance resulting from biofilms but also because of the reduced blood supply at the site of infection, which makes systemic antibiotic treatment less effective. ${ }^{1}$ Local sustained-release antibiotic systems developed in recent years are one of the most effective methods to manage chronic osteomyelitis, ${ }^{2}$ because the infectious dead space is eliminated and the concentration of local antibiotic is increased. Additionally, these systems decrease the plasma drug concentration, which in turn decreases systemic toxicity and 
side effects. However, polymethyl methacrylate (PMMA), the most common local antibiotic carrier material currently used in the clinic, has many disadvantages. These include heat production during loaded scaffold synthesis, which can result in the destruction of loaded drug and, potentially, the surrounding tissues, the requirement for surgery to remove the scaffold because it is nonabsorbable, thus increasing the chance for infection, and diminishing bone regeneration and conduction. $^{2,3}$ Therefore, antibiotic-loaded carrier fabricated with absorbable and osteogenic novel materials is of increasing interest. ${ }^{4}$ The bone-like hydroxyapatite/poly amino acid (BHA/PAA) scaffold is a novel curable biodegradable porous bone restorative material developed in our laboratory in association with Sichuan Guona Science and Technology Co. Ltd., Chengdu, People's Republic of China. ${ }^{5}$ It is polymerized in situ using BHA and PAA, which combines the favorable mechanical properties of BHA and the biocompatibility and biodegradable characteristics of PAA. By adjusting the proportion of BHA and PAA, the rate and speed of its degradation can be adjusted, suggesting that the scaffold has great potential as a bone repair and drug carrier. ${ }^{6}$ Our previous research verified that the biostructure of this scaffold was very similar to human bone in terms of its porosity and biodegradability, further supporting its potential as an ideal drug delivery carrier. Vancomycin-loaded BHA/PAA (V-BHA/ PAA) bony scaffold was successfully fabricated using a homogeneous diffusion control system. Herein, to systematically evaluate its drug-release properties and antimicrobial activity, in vitro and in vivo experiments were performed by following National Committee for Clinical Laboratory Standards guidelines, specifically for experimentation on animals. Moreover, the Ethics Committee of Chongqing Emergency Medical Center also approved this study.

\section{Materials and methods}

\section{Fabrication of V-BHA/PAA scaffold and groups}

The emulsion solvent evaporation method was used to synthesize vancomycin-encapsulated poly(lactic-co-glycolic acid) microspheres. A homogeneous method using a diffusion control system was used to incorporate vancomycinloaded poly(lactic-co-glycolic acid) microspheres into the BHA/PAA scaffold (provided by Sichuan Guona Science and Technology Co. Ltd). The final vancomycin content in the scaffold was $8 \mathrm{wt} \%$. Preparation of vancomycin-loaded PMMA (V-PMMA): $80 \mathrm{mg}$ of vancomycin powder (Eli Lilly and Company, Clinton, IN, USA) and $1 \mathrm{~mL}$ of curing liquid were added to $920 \mathrm{mg}$ of PMMA powder (DePuy Synthes
Company, West Chester, PA, USA). After thorough mixing, this cement was processed into the same size and shape as the V-BHA/PAA scaffold before curing. The final vancomycin content in V-PMMA was the same as V-BHA/PAA. For the in vitro tests, $\mathrm{V}$-BHA/PAA, V-PMMA, and BHA/PAA were all processed into disks of $6 \mathrm{~mm}$ in diameter and $1 \mathrm{~mm}$ in thickness. For the in vivo tests, materials were processed into granules of $1.5 \times 0.5 \times 0.5 \mathrm{~cm}$ and sterilized by low-temperature plasma before use. In drug-release tests, groups included the experimental group (V-BHA/PAA) and the positive control group (V-PMMA) $(n=5)$. The grouping for the in vitro antibacterial tests was carried out according to the type of infecting bacteria (regular group [regular Staphylococcus aureus group] and methicillin-resistant $S$. aureus [MRSA] group), and then subgrouped according to the type of implanted material. Each group was divided into VC (V-BHA/PAA), VUC (BHA/PAA), PC (V-PMMA), and NC (simulated body fluid $[\mathrm{SBF}]$ ) subgroups. Similarly, groups for the in vivo tests included the regular $S$. aureus group and the MRSA group, and each group was also divided into VC, PC, VUC, and NC subgroups, respectively.

\section{Culture and identification of experimental bacteria}

The regular $S$. aureus and MRSA bacterial strains were both provided by the clinical laboratory of the Fourth People's hospital in Chongqing City. The minimum inhibitory concentration (MIC) for vancomycin was determined to be $2 \mathrm{mg} / \mathrm{L}$ by the medium dilution method for both strains. After thawing, bacterial lines were cultured at $37^{\circ} \mathrm{C}$ for $24 \mathrm{~h}$. After confirming by morphological identification that all bacterial cultures were pure, they were transferred into phosphate buffer solution to prepare a bacterial suspension at $1 \times 10^{8} \mathrm{CFU} / \mathrm{mL}$.

\section{Establishment of the chronic osteomyelitis model}

Chronic osteomyelitis of tibia metaphysis in New Zealand white rabbits (provided by the Animal Experimental Center of Chongqing Medical University, Chongqing City, People's Republic of China) was induced according to the method of Lu Minpeng et al. ${ }^{7}$ The severity of osteomyelitis was graded according to gross observation and radiography scores. ${ }^{8,9}$ From those diagnosed with chronic osteomyelitis (scored at level 2 or higher), two rabbits were randomly chosen for sacrifice to harvest bone specimens. After sectioning and staining, the focus bone specimens were observed by microscopy to further confirm osteomyelitis. Moreover, bacteria culturing of the lesion marrow tissue from each rabbit was performed, and 
only the rabbits carrying the same infectious organism as the one inoculated could be used as experimental models.

\section{Drug-release test in vitro}

Samples of $105 \mathrm{mg}$ of V-BHA/PAA and V-PMMA were separately added into a $20 \mathrm{~mL}$ centrifuge tube. After adding $10 \mathrm{~mL}$ of SBF, the centrifuge tube was vibrated in a constant temperature vibrator at $30 \mathrm{rpm}$ and $37^{\circ} \mathrm{C}$. The elution was harvested every 2 days, then an additional $10 \mathrm{~mL}$ SBF was added, and the tube was continually vibrated until vancomycin could not be detected in the elution. The level of vancomycin in the elution was detected by high performance liquid chromatography (the lower detection limit was $0.2 \mathrm{mg} / \mathrm{L}$ ). At every time point, the test was repeated five times, and the average value was taken as the final value. The chromatographic conditions were as follows: analytical column $(4.6 \times 250 \mathrm{~mm}, 5 \mathrm{~mm})$, guard column (4×4 mm,5/pkg), detection wave length $236 \mathrm{~nm}$, flow velocity $1.0 \mathrm{~mL} / \mathrm{min}$, and mobile phase: $0.05 \mathrm{~mol} / \mathrm{L}$ of potassium dihydrogen phosphate solution $(\mathrm{pH}=3.2) /$ methanol $/$ acetonitrile (87:10:3). A standard curve was created using a vancomycin standard solution, and the regression analysis was carried out by plotting the concentration (C) vs peak area ratio $(\mathrm{A})(\mathrm{C}=4.75 \mathrm{~A}+0.7145[r=0.9997])$.

\section{Antibacterial zone assay}

The materials were separately placed into the center of the Müeller-Hinton agar (1.5\%) under sterile conditions just before its solidification. Vancomycin was allowed to diffuse from the materials into the agar for $4 \mathrm{~h}$ before $100 \mu \mathrm{L}$ of bacterial inoculum $\left(1 \times 10^{8} \mathrm{CFU} / \mathrm{L}\right)$ was spread over the agar plate. After the plates were incubated at $37^{\circ} \mathrm{C}$ for 5 days, the bacterial growth around the material was measured, and the diameters of the inhibition zone of the three materials were compared.

\section{Determination of bacteriostatic ratio}

The leaching solutions of each material were prepared in similar fashion to the drug-release test until the bacteriostatic ratio was lower than $50 \%$. The leaching solutions were then mixed with $4 \mathrm{~mL}$ of Müeller-Hinton broth and overnight inocula (at a final absorbance value of 0.1 at $625 \mathrm{~nm}$ ) according to the group. After incubation at $37^{\circ} \mathrm{C}$ on a shaker (50 rpm) for $12 \mathrm{~h}$, the absorbance value at $625 \mathrm{~nm}$ was measured. The bacteriostatic ratio was calculated using the following equation:

$$
\text { Bacterial inhibition }(\%)=\frac{\mathrm{Ac}-\mathrm{Ae}}{\mathrm{Ac}} \times 100 \%
$$

Ac: absorbance value of NC group; Ae: absorbance value of experimental groups. ${ }^{10}$

\section{Scanning electron microscopy (SEM)}

Disk-shaped materials were dipped into two types of bacterial suspensions (regular $S$. aureus and MRSA) at $1.5 \times 10^{6} \mathrm{CFU} / \mathrm{mL}$ and incubated at $37^{\circ} \mathrm{C}$ for $3 \mathrm{~h}$. After washing, fixation, dehydration, replacement, drying, and metal spraying, the materials with the adherent bacteria were observed by SEM.

\section{In vivo tests}

After anesthesia by intravenous injection using 3\% pentobarbital sodium and disinfection, the initial incision was reopened to expose the tibia metaphysis. Next, a bone window of $2.0 \times 1.0 \mathrm{~cm}$ was opened in the proximal tibia, followed by debridement and irritation of the infectious focus. Finally, $3 \mathrm{~g}$ of material was implanted separately into the medullary canal according to the specific group (nothing in the NC group). Postoperatively, all animals were monitored daily for activity, body temperature, weight, and appearance of the wound. Before the operation and every 2 weeks thereafter, venous blood samples were taken to determine the white blood cell (WBC) count. X-rays on the operative limbs were performed preoperatively, immediately postoperatively, and at the final time point under uniform exposure conditions to assess the progress of bone infection using Norden's radiography score. ${ }^{8}$ Additionally, two model animals from every group were chosen randomly at the preoperative time point and the final time point for bone specimen harvest from the operative area after sacrifice. The harvested bone specimens were scored on the basis of the system of Smeltzer et al, ${ }^{9}$ using microscopy of the bone section and hematoxylin and eosin staining. At the same time, bone specimens of the operative area from two additional model animals from every group were harvested under sterile conditions. After carefully removing the implants, the specimens were weighed, crunched by sterile rongeur, and ground to bone meal by mortar, followed by homogenization for 5 min using a T-25 homogenizer (10,000 rpm) after adding $10 \mathrm{~mL}$ of phosphate buffer solution per $100 \mathrm{~g}$ bone meal. The homogenate of every model animal was harvested and diluted ten times, and a $0.1 \mathrm{~mL}$ dilution was spread on a blood agar plate. After incubating for $48 \mathrm{~h}$ at $37^{\circ} \mathrm{C}$, the colonies were counted, and the bacterial count per $1 \mathrm{~g}$ of bone specimen was calculated. For statistical analysis, the negative culture was determined to be $2 \times 10^{3} \mathrm{CFU} / \mathrm{g}$ (corresponding to the limit of detection). 


\section{Statistical analysis}

Data were analyzed using the Statistical Package for the Social Sciences (SPSS) version 11.0 (SigmaStat; SPSS Inc., Chicago, IL, USA), and the results were reported as the mean \pm standard deviation. An independent sample $t$-test was used to compare the diameter of the antibacterial ring and vancomycin content between groups. A one-way analysis of variance (ANOVA) test was used to compare body weight, WBC count, scores of X-ray, and histology. In addition, Kruskal-Wallis $H$ test was applied to assess differences in bacterial count between groups. Significant differences had $P$-values of less than 0.05 .

\section{Results}

\section{Drug-release test}

As shown in Figure 1, during the first $48 \mathrm{~h}$, vancomycin in either the PC group or the VC group was released relatively rapidly, followed by a period of slow and sustained release. More specifically, in vitro release of vancomycin from the V-BHA/PAA scaffold was a three-phase process with a second release peak on day 20. The release of vancomycin from V-PMMA was characterized by an early burst release without the second release peak. As shown in Figure 2, the cumulative amount of vancomycin released from V-BHA/PAA during the first $48 \mathrm{~h}$ was $43.02 \%$, increasing to $89.22 \%$ on day 38 , after which nothing was detected (the lower detection limit was $0.2 \mathrm{mg} / \mathrm{L}$ ). In total, there were 30 days when the concentration of vancomycin stayed above its MIC for S. aureus $\left(\mathrm{MIC}_{90}=1 \mathrm{mg} / \mathrm{L}\right)$. Correspondingly, the amount of vancomycin release from V-PMMA during the first $48 \mathrm{~h}$ and at the final time point was $41.15 \%$ and $86.86 \%$, respectively, and not significantly different from V-BHA/PAA $(P=0.621$, $P=0.133$ ), although its duration of sustained release above the $\mathrm{MIC}_{90}$ (18 days) was significantly shorter than that of V-BHA/PAA $(P=0.005, P=0.015)$.

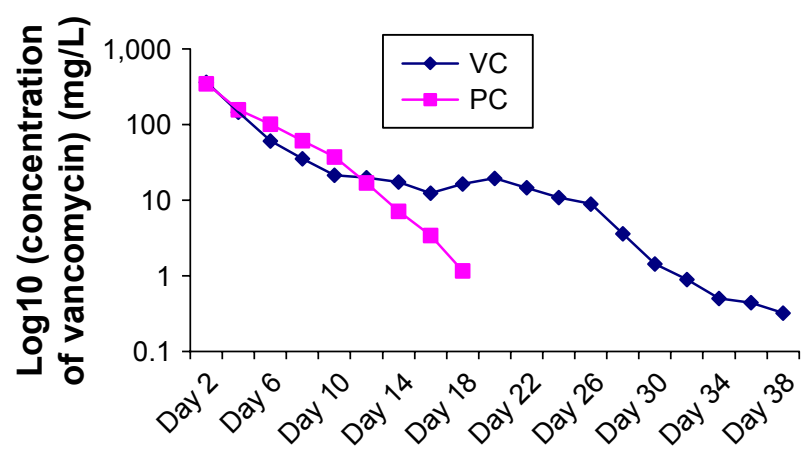

Figure I Release curve of vancomycin in vitro.

Abbreviations: VC, vancomycin-loaded bone-like hydroxyapatite/poly amino acid group; PC, vancomycin-loaded polymethyl methacrylate group.

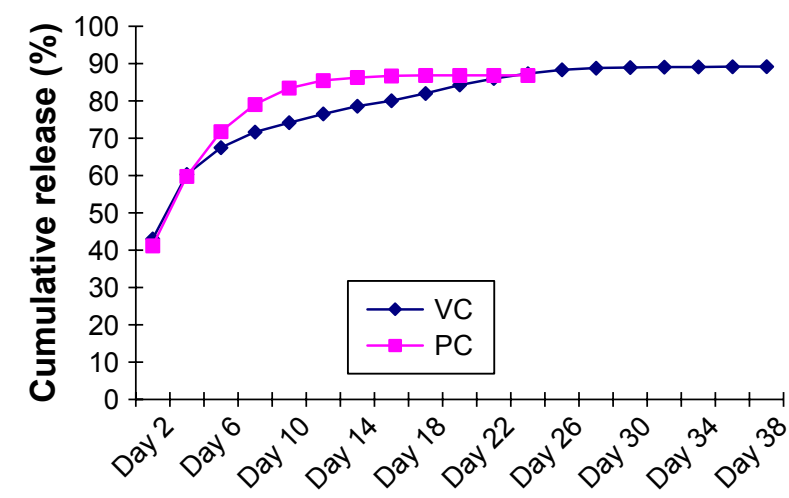

Figure 2 Cumulative release percentage of vancomycin.

Abbreviations: VC, vancomycin-loaded bone-like hydroxyapatite/poly amino acid group; PC, vancomycin-loaded polymethyl methacrylate group.

\section{Antibacterial zone assay}

As shown in Figure 3, the agar plates containing the BHA/ PAA scaffold were completely covered with bacteria after 5 days of incubation. In contrast, the growth of $S$. aureus and that of MRSA were both inhibited in the regions around the V-BHA/PAA and V-PMMA materials. However, as shown in Table 1, the diameter of the zone of inhibition on the plates with V-BHA/PAA was much larger than that with V-PMMA $(P<0.05)$.

\section{Determination of bacteriostatic ratio}

The GB-15979 guideline states that material with an inhibition of bacterial growth $\geq 50 \%$ can be regarded as having antibacterial activity, while material with an inhibition of bacterial growth $\geq 90 \%$ can be regarded as having excellent antibacterial activity. The antibacterial activity of V-BHA/ PAA toward the two bacterial strains lasted for more than 28 days (51.59\% of regular S. aureus and $50.91 \%$ of MRSA growth was inhibited on day 28). The antibacterial activity of V-PMMA decreased rapidly after 12 days and lasted for just 14 days $(61.68 \%$ of regular S. aureus and $54.74 \%$ of MRSA
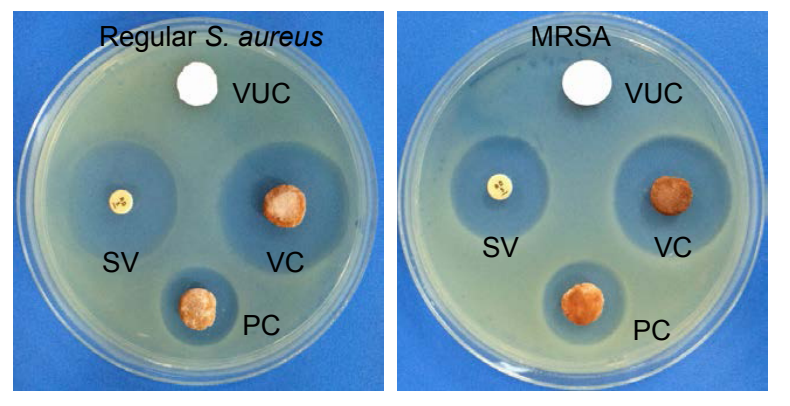

Figure 3 Whether on regular S. aureus or MRSA, VC subgroup both showed strongest antibacterial activity.

Abbreviations: MRSA, methicillin-resistant Staphylococcus aureus; VUC, bone-like hydroxyapatite/poly amino acid group; PC, vancomycin-loaded polymethyl methacrylate group; SV, standard vancomycin; VC, vancomycin-loaded bone-like hydroxyapatite/ poly amino acid group. 
Table I Diameter of bacterial inhibition zone $(\overline{\mathrm{x}} \pm \mathrm{SD}$, unit: $\mathrm{mm})$

\begin{tabular}{llll}
\hline Bacterial strains & VC & PC & VUC \\
\hline Regular S. aureus & $25.49 \pm 2.58$ & $11.82 \pm 1.38$ & 0 \\
MRSA & $27.93 \pm 3.52$ & $13.96 \pm 2.42$ & 0 \\
\hline
\end{tabular}

Notes: Regular $S$. aureus: VC group vs $P C$ group, $P=0.002$; MRSA: VC group vs $P C$ group, $P=0.009$.

Abbreviations: SD, standard deviation; MRSA, methicillin-resistant Staphylococcus aureus; VUC, bone-like hydroxyapatite/poly amino acid group; PC, vancomycinloaded polymethyl methacrylate group; VC, vancomycin-loaded bone-like hydroxyapatite/poly amino acid group.

growth was inhibited on day 14). The BHA/PAA scaffold did not provide any antibacterial effects, providing less than $20 \%$ inhibition of growth of either bacteria (Figures 4 and 5).

\section{Observation of bacterial colonization by SEM}

As shown in Figures 6 and 7, bacteria were observed adhering as lumps or in a linear distribution to the surface of the BHA/PAA material by SEM in both the regular $S$. aureus and MRSA groups. However, fewer bacteria were observed on the surface of V-BHA/PAA and V-PMMA, presenting as a diffused distribution.

\section{General observations from the in vivo tests}

There were two animals in subgroups VUC and $\mathrm{NC}$ of the regular $S$. aureus group, one animal in subgroup PC, and two animals in subgroup NC of the MRSA group that died from severe infection, as confirmed by autopsy. The remaining animals recovered from surgery over the course of the experiment. One animal in subgroup $\mathrm{NC}$ of the regular $S$. aureus group and one in subgroup VUC of the MRSA group were observed to have a pathologic fracture in the operation area. The body weight of all animals consistently decreased over the first 2 weeks after debridement. After

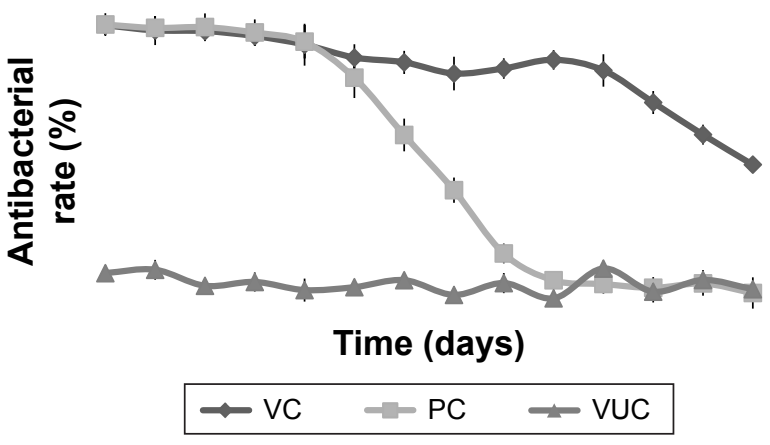

Figure 4 Antibacterial rate curve on regular $S$. aureus.

Note: Each data point represents a mean \pm standard deviation $(n=5)$.

Abbreviations: S. aureus, Staphylococcus aureus; VUC, bone-like hydroxyapatite/ poly amino acid group; PC, vancomycin-loaded polymethyl methacrylate group; VC, vancomycin-loaded bone-like hydroxyapatite/poly amino acid group.

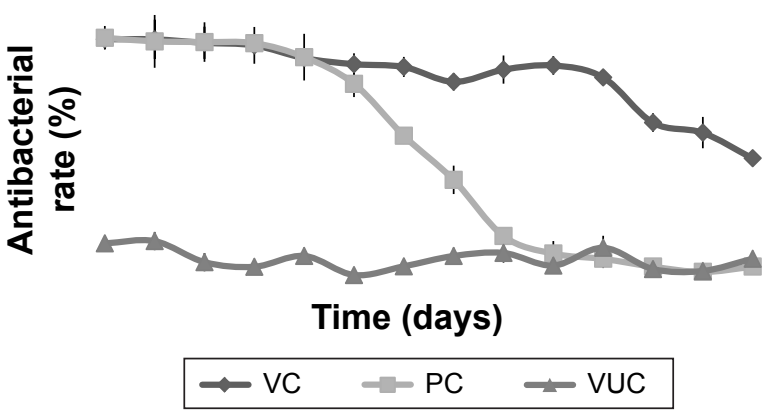

Figure 5 Antibacterial rate curve on MRSA.

Note: Each data point represents a mean \pm standard deviation $(n=5)$.

Abbreviations: MRSA, methicillin-resistant Staphylococcus aureus; VUC, bone-like hydroxyapatite/poly amino acid group; PC, vancomycin-loaded polymethyl methacrylate group; VC, vancomycin-loaded bone-like hydroxyapatite/poly amino acid group.

this time, the body weight of animals from subgroups VC and PC of both bacterial strains increased continuously until at 8 weeks postsurgery, they exceeded their initial weight. However, the body weight of animals from subgroups VUC and NC decreased throughout the entire course of the experiment. At the end of the experiment, the mean body weight had increased significantly in subgroups $\mathrm{VC}$ and $\mathrm{PC}$ of both bacterial strains and significantly decreased in subgroups VUC and NC (Figure 8). Although the body temperatures fluctuated after every operation, they were not significantly different at the time of sacrifice compared with the preoperative levels within a group or among groups (data not shown).

\section{Venous WBC count}

As shown in Figure 9, the WBC count in every group increased gradually during the first 2 weeks, followed by a decrease in subgroups $\mathrm{VC}$ and $\mathrm{PC}$ of the two groups, ultimately falling below the level at pretreatment. However, the WBC count in subgroups VUC and NC of the two groups continued to rise so that the final count was significantly higher than the preoperative level.

\section{Radiographic performance}

All or some of the typical signs of chronic osteomyelitis, including destruction of bone, sequestral bone formation, periosteal reaction, bone sclerosis, and soft tissue swelling, were observed radiographically in every group before the treatment operation (Figure 10). At postoperative week 6, varying degrees of elimination of bone infection were observed on the X-ray plates of the two bacteria groups (Figure 10). When we compared the extent of elimination of infection between the two bacteria groups, subgroups $\mathrm{VC}$ and $\mathrm{PC}$ were significantly more improved than subgroups 

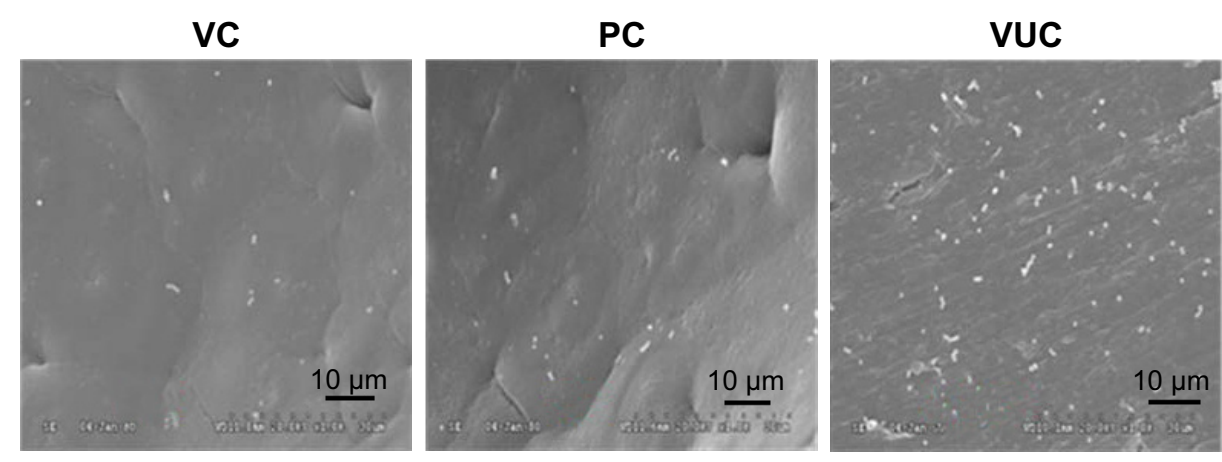

Figure 6 Adhesion of regular S. aureus on the surface of scaffolds under SEM $\times$ I k.

Abbreviations: SEM, scanning electron microscopy; S. aureus, Staphylococcus aureus; VUC, bone-like hydroxyapatite/poly amino acid group; PC, vancomycin-loaded polymethyl methacrylate group; VC, vancomycin-loaded bone-like hydroxyapatite/poly amino acid group.

VUC and NC, and subgroup VC was more improved than subgroup PC (Table 2). As shown in Figure 11, compared with pretreatment, the Norden's osteomyelitis scores of subgroups VC and PC from the two bacterial groups were both significantly improved, while those of subgroups VUC and NC did not improve. Subgroup VC from both bacterial groups showed the best improvement, subgroup PC the second best, while a comparison of subgroups VUC and NC showed no significant difference.

\section{Histological characteristics}

As shown in Figure 12, the histological characteristics of both the regular $S$. aureus and MRSA groups were comparable. Subgroup VC of the two bacterial groups exhibited mild infiltration of inflammatory cells and fibrosis accompanied by new bone formation located at the interface of the material and bone. No obvious new bone formation was observed in subgroup PC, although infiltration of inflammatory cells and fibrosis was more obvious than in subgroup VC. Various degrees of osteomyelitis features, such as inflammatory reaction of bone or periosteum and bone necrosis, were exhibited in subgroups VUC and NC. A large number of neutrophils or mononuclear cells and significant fibrosis were observed surrounding the materials without obvious new bone formation, indicating that a serious inflammatory response would restrain bone regeneration. As shown in Figure 13, the histological scores of the corresponding subgroups of the two bacterial strains were not significantly different. The scores of subgroups VC and PC at week 12 after treatment were considerably below those at pretreatment, while those of subgroups VUC and NC showed no obvious differences. A comparison of the Norden scores among the subgroups at the end of treatment indicated subgroup $\mathrm{VC}<$ subgroup $\mathrm{PC}<$ subgroup VUC or NC, with no difference between subgroups VUC and NC.

\section{Microbiological characterization}

The best evidence of infection elimination was obtained from the microbiological inspection. In this test, no bacteria could be cultured from 9 out of 12 animals from regular subgroup VC and 6/12 from subgroup PC, which was significantly higher than other subgroups of this group (regular-VUC: 3/12, regular-NC: $2 / 12$ ). Likewise, in the MRSA group, $8 / 12$ or $5 / 12$ animals of the VC or PC subgroup exhibited negative culture, which was significantly higher than in other subgroups (MRSA-VUC: 2/12, MRSA-NC: 2/12). The bacterial load per gram of bone is plotted in Figure 14. As shown, bacterial load per gram of bone between the two
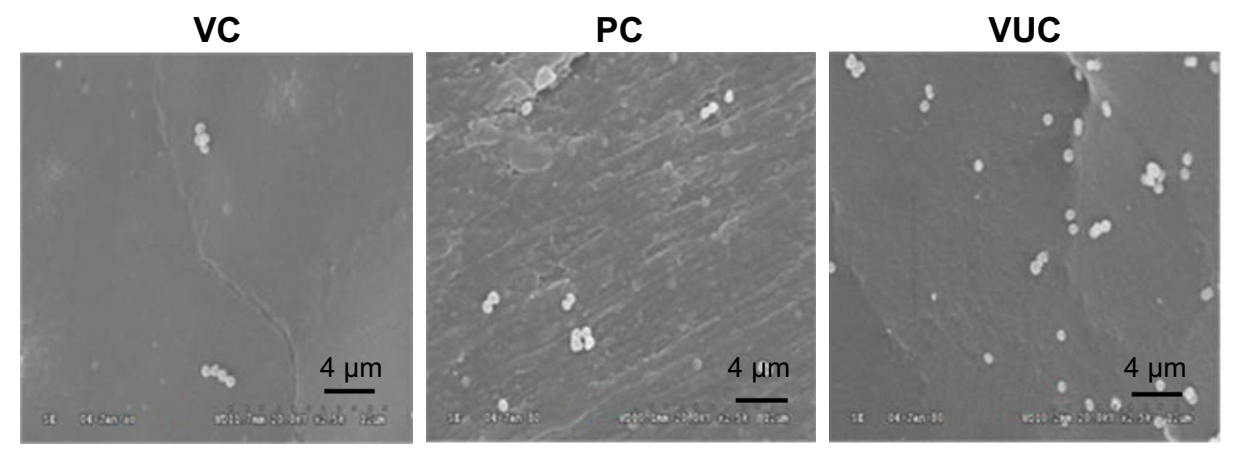

Figure 7 Adhesion of MRSA on the surface of scaffolds under SEM $\times 2 \mathrm{k}$.

Abbreviations: MRSA, methicillin-resistant Staphylococcus aureus; SEM, scanning electron microscopy; VUC, bone-like hydroxyapatite/poly amino acid group; PC, vancomycinloaded polymethyl methacrylate group; VC, vancomycin-loaded bone-like hydroxyapatite/poly amino acid group. 


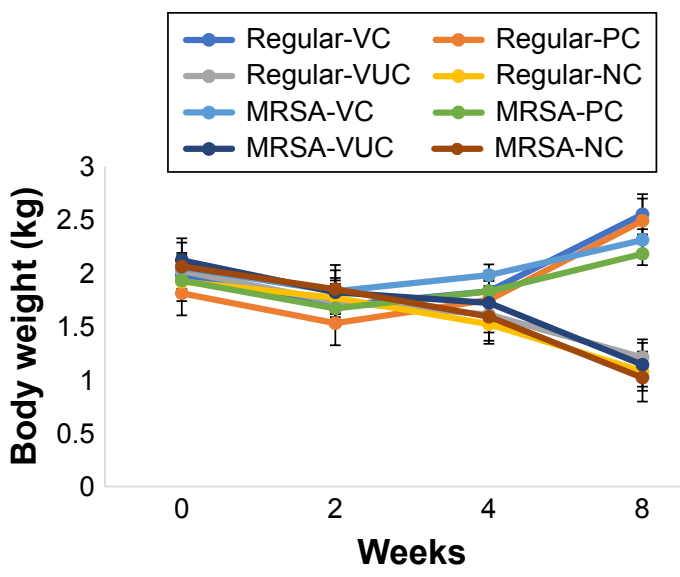

Figure 8 The change of body weight.

Notes: Regular-VC referred to subgroup VC of regular S. aureus group, and so on. Comparison of weight between pretreatment and the end time: regular-VC: $P=0.007$; regular- $P C$ : $P=0.09$; regular-VUC: $P=0.02$; regular-NC: $P=0.04$; $M R S A-V C$ : $P=0.01$; MRSA-PC: $P=0.08$; MRSA-VUC: $P=0.04$; MRSA-NC: $P=0.02$.

Abbreviations: MRSA, methicillin-resistant Staphylococcus aureus; VC, vancomycinloaded bone-like hydroxyapatite/poly amino acid group; VUC, bone-like hydroxyapatite/poly amino acid group; PC, vancomycin-loaded polymethyl methacrylate group; NC, simulated body fluid group.

bacterial strains have no significant difference. However, significantly lower bacterial load was shown in subgroups $\mathrm{VC}$ and $\mathrm{PC}$ from the two groups than prior treatment level $(P<0.01)$, while that of subgroups VUC and NC showed no difference $(P>0.05)$. The results of comparison among subgroups at the end of treatment were as follows: subgroup $\mathrm{VC}<$ subgroup $\mathrm{PC}<$ subgroup VUC or $\mathrm{NC}$, and no difference between subgroup VUC and NC.

\section{Discussion}

Chronic osteomyelitis is often secondary to open fracture, bacteremia, and adjacent soft tissue infection. The incidence

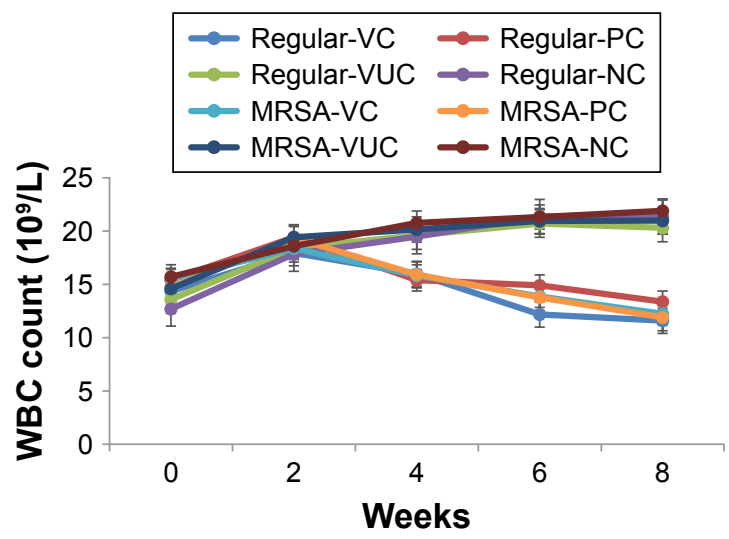

Figure 9 The change of venous WBC count.

Notes: Regular-VC referred to subgroup VC of regular S. aureus group, and so on. Comparison of WBC count between pretreatment and the end time: regular-VC: $P=0.03$; regular $-P C$ : $P=0.04$; regular-VUC: $P=0.008$; regular-NC: $P=0.005$; MRSA-VC: $P=0.02$; MRSA-PC: $P=0.04$; MRSA-VUC: $P=0.006$; MRSA-NC: $P=0.006$.

Abbreviations: WBC, white blood cell; MRSA, methicillin-resistant Staphylococcus aureus; VC, vancomycin-loaded bone-like hydroxyapatite/poly amino acid group; VUC, bone-like hydroxyapatite/poly amino acid group; PC, vancomycin-loaded polymethyl methacrylate group; NC, simulated body fluid group. of bone infection followed by open fracture can approach $27 \% .{ }^{11}$ Along with increasing incidents of diabetic foot infection and peripheral vascular disease, chronic osteomyelitis resulting from adjacent soft tissue has become increasingly common. ${ }^{12}$ The most typical pathogen involved in adult bone and joint infection is $S$. aureus. In recent years, a growing number of MRSA were cultured from osteomyelitis patients. In fact, more than one-third of the isolated $S$. aureus strains from adult infectious bones were identified as MRSA. ${ }^{13}$ In clinic, diagnosis of chronic osteomyelitis includes typical manifestation of the condition as well as physical examination, lab and radiographic detection, of which microbial culture of the infection is the most valuable. Positive results of bone biopsy associated with typical necrotic histopathology are generally recognized as the gold standard for diagnosing chronic osteomyelitis. ${ }^{14}$ In this study, only animals with bone biopsy results in line with the initially inoculated bacteria and typical histological characteristics were chosen as models for further study.

Surgical debridement and prolonged parenteral antibiotic administration constitute the current main therapeutic measures to treat chronic osteomyelitis. However, the recurrence rate is high, reportedly up to $30 \%$ at 12 months after surgery. ${ }^{15}$ The involvement of biofilm in the infection is related to the diversity of clinical symptoms observed in chronic osteomyelitis and the variability in the course of the disease. ${ }^{16}$ Generally, necrotic tissue including sequestrum induces the formation of biofilm because necrotic tissue takes the form of a foreign body in vivo and becomes infected by bacteria. Initially, the parasitic bacteria colonize the surface of the necrotic tissue, and then enter into the three-dimensional structure of the necrotic tissue and undergo geometric proliferation. The bacteria can communicate with each other via autoinduction (chemical signals) either within or between bacterial communities. ${ }^{17}$ In this way, a defensive barrier is formed to protect the bacteria from the attack of the host immune system and antibiotic treatment. Therefore, to cure chronic osteomyelitis, similar to the treatment for cancer, it is often necessary to radically excise the infected area together with the surrounding healthy bone and soft tissues. ${ }^{18}$ However, generally surgical debridement and excision are not sufficient, and antibiotics must also be administered. ${ }^{19}$ Because bacteria can still be present inside the dead spaces and defects after debridement, recurrence of infection is possible if antibiotic treatment is not persistent enough to kill the residual bacteria. In this study, in the two control groups (subgroups VUC and NC) that were debrided without antibiotic treatment, the recurrence rate of infection was more than $70 \%$ at 12 weeks postsurgery. 

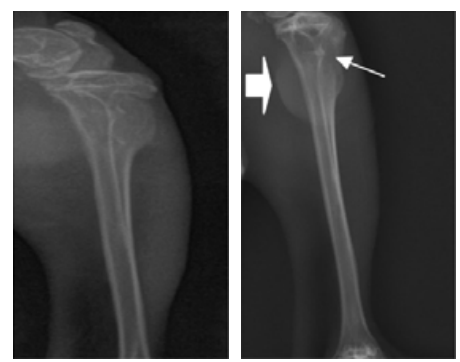

Regular-Pre

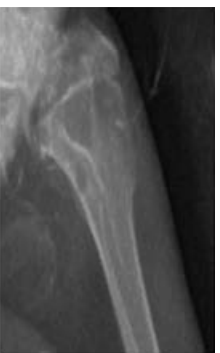

MRSA-Pre

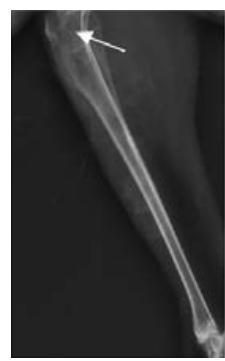

MRSA-NC
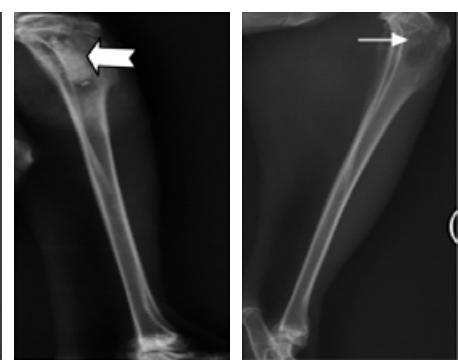

Regular-PC

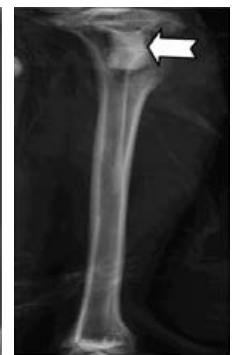

MRSA-VUC

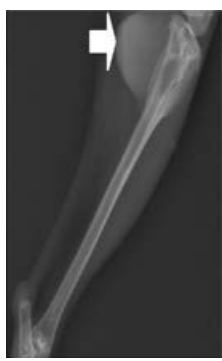

MRSA-PC

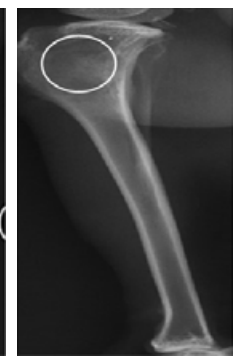

Regular-VC

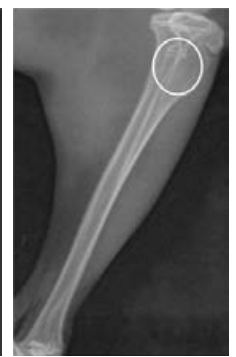

MRSA-VC

Figure 10 Radiographic performance.

Notes: Regular-Pre means presurgery in the regular S. aureus group; the solid arrow $(\longrightarrow$ ) represents a bone defect; the arrowhead ( $\Rightarrow$ ) represents reactive ossification; the swallowtail arrowhead $(\longleftrightarrow)$ represents the enlarged medullary cavity; the circle represents bone healing.

Abbreviations: MRSA, methicillin-resistant Staphylococcus aureus; VC, vancomycin-loaded bone-like hydroxyapatite/poly amino acid group; VUC, bone-like hydroxyapatite/ poly amino acid group; PC, vancomycin-loaded polymethyl methacrylate group; NC, simulated body fluid group.

Vancomycin, a glycopeptide antibiotic, is administered primarily to prevent and treat the infection caused by gram-positive bacteria, especially drug-resistant strains. ${ }^{20}$ The increase of drug-resistant bacteria including MRSA involved in chronic osteomyelitis has led to the use of vancomycin as the first-line antibiotic of choice to treat chronic osteomyelitis in the clinic. However, vancomycin is mainly administered systemically by intravenous administration in the clinic, which can cause severe nephrotoxicity and ototoxicity. In addition, the lack of blood supply to the infectious area limits the amount of antibiotic that can reach the site, potentially decreasing the beneficial effect contributing to drug-resistant bacteria. ${ }^{21}$ Instead, local sustained antibiotic delivery systems have many advantages. The side and toxic

Table 2 The number of bone infection elimination cases (unit: cases, $n=12$ )

\begin{tabular}{lll}
\hline Groups & Regular S. aureus group & MRSA group \\
\hline Subgroup VC & 9 & 8 \\
Subgroup PC & 6 & 5 \\
Subgroup VUC & 1 & 0 \\
Subgroup NC & 1 & 0 \\
\hline
\end{tabular}

Notes: Elimination of bone infection defined as disappearance of typical infectious signs on X-ray. Regular-VC vs regular-PC and MRSA-VC vs MRSA-PC: $P=0.03$, regular-VC vs regular-VUC, regular-VC vs regular-NC, MRSA-VC vs MRSA-VUC and MRSA-VC vs MRSA-NC: $P=0.003$; regular-PC vs regular-VUC, regular-PC vs regular-NC, MRSA-PC vs MRSA-VUC and MRSA-PC vs MRSA-NC: $P=0.008$; regular-VUC vs regular-NC and MRSA-VUC vs MRSA-NC: $P=0.09$.

Abbreviation: MRSA, methicillin-resistant Staphylococcus aureus. effects of the antibiotic are decreased because the blood concentration is lowered, while local antibiotic delivery can maintain a relatively high concentration for a longer time..$^{22}$ Furthermore, local sustained release of antibiotics contributes

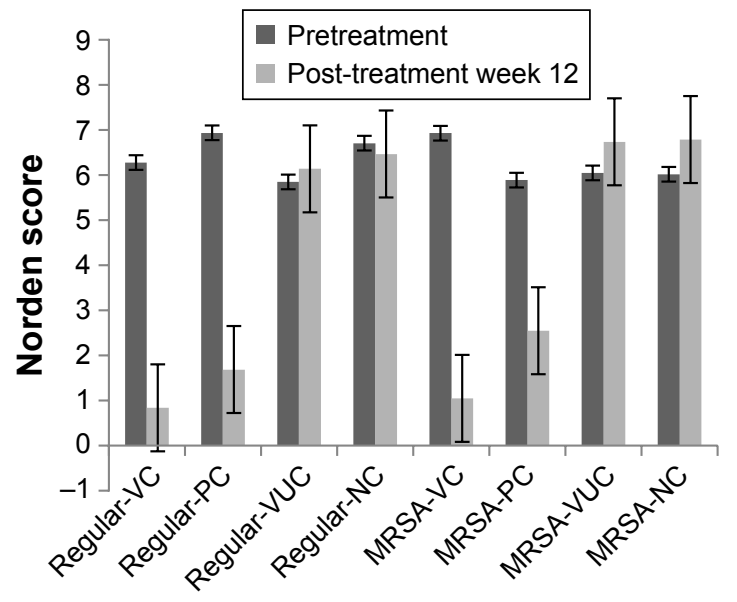

Figure I I Radiographic score before and after treatment.

Notes: Comparison of Norden scores between before and after treatment: regular-VC: $P=0.003$, regular-PC: $P=0.003$, regular-VUC: $P=0.10$, regular-NC: $P=0.1$, MRSA-VC: $P=0.005$, MRSA-PC: $P=0.009$, MRSA-VUC: $P=0.14$, MRSA-NC: $P=0.12$; Comparison among subgroups after treatment: regular-VC vs regular-PC and MRSA-VC vs MRSA-PC: $P=0.03$; regular-VC vs regular-VUC, regular-VC vs regular-NC, MRSA-VC vs MRSA-VUC and MRSA-VC vs MRSA-NC: $P=0.003$; regular-PC vs regular-VUC, regular-PC vs regular-NC, MRSA-PC vs MRSA-VUC and MRSA-PC vs MRSA-NC: $P=0.007$; regular-VUC vs regular-NC and MRSA-VUC vs MRSA-NC: $P=0.08$, No statistical difference in corresponding subgroups between regular group and MRSA group $(P>0.05)$.

Abbreviations: MRSA, methicillin-resistant Staphylococcus aureus; VC, vancomycinloaded bone-like hydroxyapatite/poly amino acid group; VUC, bone-like hydroxyapatite/poly amino acid group; PC, vancomycin-loaded polymethyl methacrylate group; NC, simulated body fluid group. 

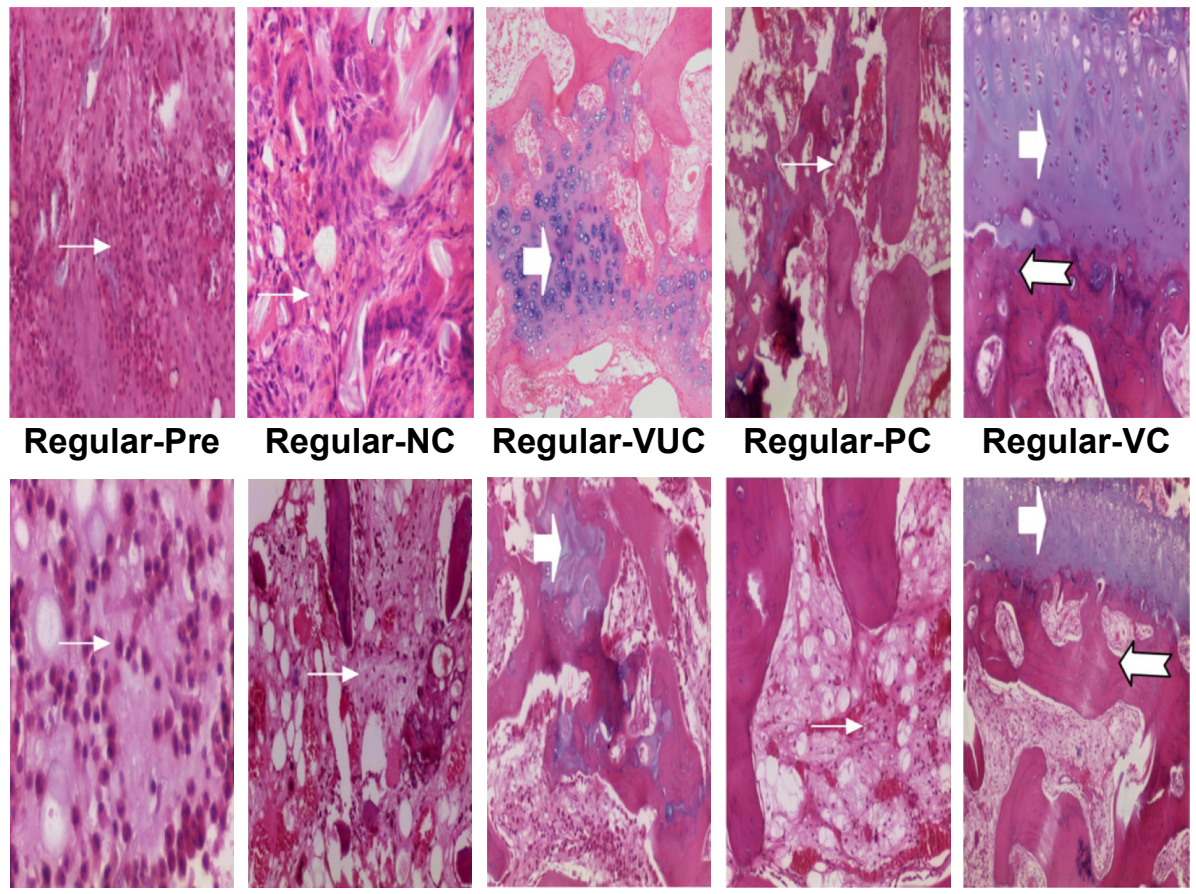

MRSA-Pre

MRSA-NC

MRSA-VUC

MRSA-PC

MRSA-VC

Figure 12 Histological performance (H\&E staining, $\times 100)$.

Notes: Regular-Pre means presurgery in the regular S. aureus group; the solid arrow $(\longrightarrow)$ indicates inflammatory cell infiltration; the arrowhead $(\Longrightarrow)$ indicates new chondrogenesis; the swallowtail arrowhead $(\longleftarrow)$ indicates new trabecular bone formation. Magnification $\times 100$.

Abbreviations: H\&E, hematoxylin and eosin; MRSA, methicillin-resistant Staphylococcus aureus; VC, vancomycin-loaded bone-like hydroxyapatite/poly amino acid group; VUC, bone-like hydroxyapatite/poly amino acid group; PC, vancomycin-loaded polymethyl methacrylate group; NC, simulated body fluid group.

to decreasing the appearance of drug-resistant organisms by improving the bioavailability of antibiotics through siteand time-specific delivery. ${ }^{23}$ The vancomycin-loaded BHA/ PAA scaffold fabricated by our group has the capacity to persistently release drugs at the desired site. The drug delivery testing demonstrated that V-BHA/PAA could stably release loaded vancomycin in vitro and in vivo. Over the first $48 \mathrm{~h}$, vancomycin was released more rapidly, followed by a period of sustained slow release. The period of early rapid release helps to immediately increase the local drug concentration so that most of the pathogens are killed as quickly as possible. The period of sustained delivery helps to maintain a local effective bactericidal concentration for a prolonged period of time to kill the residual pathogens. Because of its three-phase delivery feature with two release peaks, V-BHA/PAA had relatively long-term vancomycin delivery, 38 days in vitro and

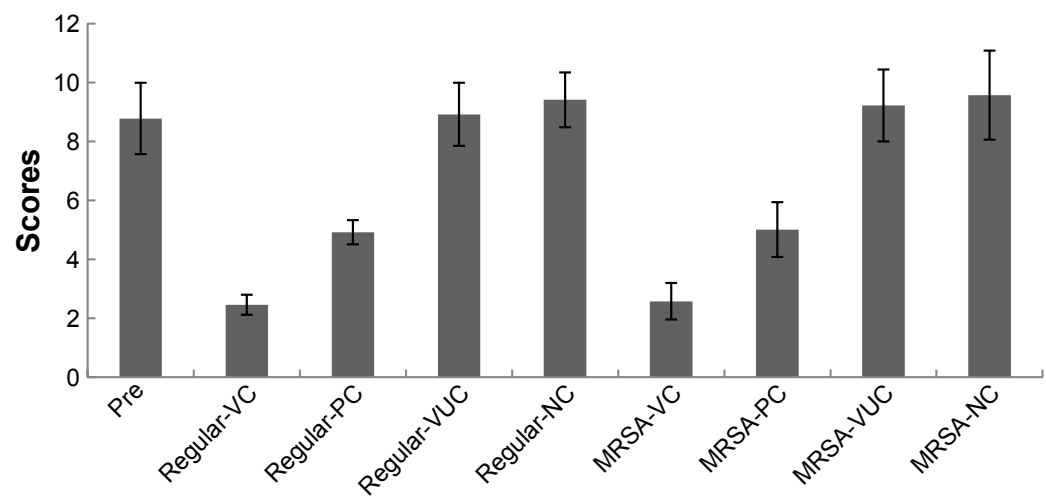

Figure 13 Histological score before and after treatment.

Notes: Pre means pretreatment. Comparison of histological scores between before and after treatment: regular-VC: $P<0.00 \mathrm{I}$, regular-PC: $P=0.03$, regular-VUC: $P=0.14$, regular-NC: $P=0.16$, MRSA-VC: $P<0.001$, MRSA-PC: $P=0.02$, MRSA-VUC: $P=0.31$, MRSA-NC: $P=0.52$; Comparison among subgroups after treatment: regular-VC vs regular$P C$ and MRSA-VC vs MRSA-PC: $P=0.03$; regular-VC vs regular-VUC, regular-VC vs regular-NC, MRSA-VC vs MRSA-VUC and MRSA-VC vs MRSA-NC: $P=0.009$; regular-PC vs regular-VUC, regular-PC vs regular-NC, MRSA-PC vs MRSA-VUC and MRSA-PC vs MRSA-NC: $P=0.04$; regular-VUC vs regular-NC and MRSA-VUC vs MRSA-NC: $P=0.17$, No statistical difference in corresponding subgroups between regular group and MRSA group $(P>0.05)$.

Abbreviations: MRSA, methicillin-resistant Staphylococcus aureus; VC, vancomycin-loaded bone-like hydroxyapatite/poly amino acid group; VUC, bone-like hydroxyapatite/ poly amino acid group; PC, vancomycin-loaded polymethyl methacrylate group; NC, simulated body fluid group. 


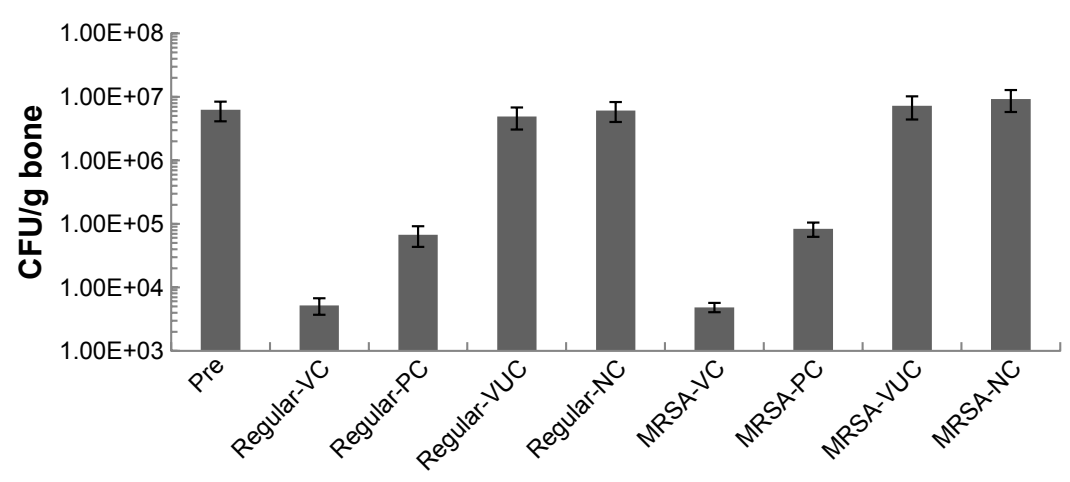

Figure I 4 Bacterial load per gram bone.

Notes: Pre means pretreatment. Comparison of bacterial load between before and after treatment: regular-VC: $P<0.00 \mathrm{I}, \mathrm{regular}-\mathrm{PC}: P=0.04$, regular-VUC: $P=0.2 \mathrm{I}$, regularNC: $P=0.35$, MRSA-VC: $P<0.00$ I, MRSA-PC: $P=0.03$, MRSA-VUC: $P=0.19$, MRSA-NC: $P=0.16$; Comparison among subgroups after treatment: regular-VC vs regular-PC and MRSA-VC vs MRSA-PC: $P=0.03$; regular-VC vs regular-VUC, regular-VC vs regular-NC, MRSA-VC vs MRSA-VUC and MRSA-VC vs MRSA-NC: $P=0.006$; regular-PC vs regular-VUC, regular-PC vs regular-NC, MRSA-PC vs MRSA-VUC and MRSA-PC vs MRSA-NC: $P=0.03$; regular-VUC vs regular-NC and MRSA-VUC vs MRSA-NC: $P=0.15$, No statistical difference in corresponding subgroups between regular group and MRSA group $(P>0.05)$.

Abbreviations: MRSA, methicillin-resistant Staphylococcus aureus; VC, vancomycin-loaded bone-like hydroxyapatite/poly amino acid group; VUC, bone-like hydroxyapatite/ poly amino acid group; PC, vancomycin-loaded polymethyl methacrylate group; NC, simulated body fluid group.

42 days in vivo, which corresponded with the requirements of antibiotics used to treat chronic osteomyelitis (4-6 weeks).

To systematically evaluate the antibacterial functions of V-BHA/PAA as well as the influence of processing the local in vivo environment on its antimicrobial activity, antibacterial tests in vitro and in vivo were designed. The results demonstrated that $\mathrm{V}$-BHA/PAA showed significant and consistent bactericidal effects in vitro and in vivo toward both regular $S$. aureus and MRSA. Moreover, the bactericidal effect of V-BHA/PAA was obviously stronger than that of V-PMMA, the most commonly used local antibiotic delivery system in the clinic to date. Based on the GB-15979 guideline, the antibacterial duration of V-BHA/PAA toward regular $S$. aureus or MRSA exceeded 28 days in vitro, while that of V-PMMA lasted only 14 days. The duration and amount of persistent vancomycin delivery from the V-BHA/PAA scaffold were both far superior to those of V-PMMA. The antibacterial curve of V-BHA/PAA in vitro was found to almost coincide with its vancomycin delivery curve, which indicated that the BHA/PAA material could control the delivery of vancomycin to prolong the duration of its antibacterial action, but also effectively maintain the antibacterial activity of the loaded vancomycin. Likewise, in vivo treatment with V-BHA/PAA was effective at treating chronic osteomyelitis generated by regular $S$. aureus and MRSA. After treatment by debridement and implantation of V-BHA/PAA scaffold, the infection index of each animal, including their general condition, blood WBC count, radiography, and histology, showed improvements over the control groups. Furthermore, local bacterium quantitation of the V-BHA/PAA treated group also revealed that almost all infection was eliminated. Ultimately, the curative ratio of chronic osteomyelitis after treatment with the V-BHA/PAA scaffold reached $75 \%$ for regular $S$. aureus and $66.67 \%$ for MRSA, which significantly exceeded that of V-PMMA (50\% and 41.67\%, respectively).

\section{Conclusion}

The vancomycin released from the V-BHA/PAA scaffold, a novel vancomycin-loaded bone repair material, was an effective antibacterial agent in vitro and in vivo toward both regular $S$. aureus and MRSA. Moreover, the antibacterial activity of the V-BHA/PAA scaffold was significantly superior to V-PMMA. Its biodegradability and inductive osteogenesis potentially make V-BHA/PAA an ideal option for treating chronic bone infection. ${ }^{24}$ Nevertheless, in this study, we also determined that this protocol of debridement combined with implantation of the V-BHA/PAA scaffold could not completely cure every case, implying that it would be necessary to include other treatments, such as systemic antibiotics. In addition, the long-term effects and risks, when PMMA was removed or V-BHA/PAA was allowed, were not assessed. Therefore, further work is required to expand upon this research.

\section{Acknowledgments}

All authors were particularly grateful to NSFC (the National Natural Science Foundation of China) for financial support (No 81171685) and to the clinical laboratory of the Fourth People's hospital in Chongqing City for donating bacterial strains.

\section{Disclosure}

The authors report no conflicts of interest in this work. 


\section{References}

1. Brady RA, Leid JG, Costerton JW, Shirtliff ME. Osteomyelitis: clinical overview and mechanisms of infection persistence. Clin Microbiol Newsl. 2006;28:65-72.

2. Zilberman M, Elsner JJ. Antibiotic-eluting medical devices for various applications. J Control Release. 2008;130:202-215.

3. Kluin OS, van der Mei HC, Busscher HJ, Neut D. Biodegradable vs non-biodegradable antibiotic delivery devices in the treatment of osteomyelitis. Expert Opin Drug Deliv. 2013;10(3):341-351.

4. Giavaresi G, Bertazzoni Minelli E, Sartori M, et al. Microbiological and pharmacological tests on new antibiotic-loaded PMMA-based composites for the treatment of osteomyelitis. J Orthop Res. 2012; 30(3):348-355.

5. Dai Zhenyu, Li Jun, Zhao ZH, Ao XX, Jiang DM. Biological safety of nano-hydroxyapatite and poly-amino acid compound. J Third Mil Med Univ. 2010;32(2):2294-2299.

6. Huang J, Zhao D, Dangaria SJ, et al. Combinatorial design of hydrolytically degradable, bone-like biocomposites based on PHEMA and hydroxyapatite. Polymer (Guildf). 2013;54(2):909-919.

7. Lu Minpeng, Jiang Dianming, Quan Zhengxue, et al. The establishment of experimental chronic osteomyelitis induced by Staphylococcus aureus in rabbits. Chinese J Exp Surg. 2010;27(2):110-113.

8. Norden CW, Myerowitz RL, Keleti E. Experimental osteomyelitis due to Staphylococcus aureus or Pseudomonas aeruginosa: a radiographicpathological correlative analysis. Br J Exp Pathol. 1980;61:451-460.

9. Smeltzer MS, Thomas JR, Hickmon SG, et al. Characterization of a rabbit model of staphylococcal osteomyelitis. J Orthop Res. 1997;15: 414-421.

10. Yin LY, Lazzarini L, Li F, et al. Comparative evaluation of tigecycline and vancomycin, with and without rifampicin, in the treatment of methicillinresistant Staphylococcus aureus experimental osteomyelitis in a rabbit model. J Antimicrob Chemother. 2005;55(6):995-1002.

11. Pollak AN, Jones AL, Castillo RC, Bosse MJ, MacKenzie EJ; LEAP Study Group. The relationship between time to surgical debridement and incidence of infection after open high-energy lower extremity trauma. J Bone Joint Surg Am. 2010;92(1):7-15.

12. Walter G, Kemmerer M, Kappler C, Hoffmann R. Treatment algorithms for chronic osteomyelitis. Dtsch Arztebl Int. 2012;109(14):257-264.
13. Hatzenbuehler J, Pulling TJ. Diagnosis and management of osteomyelitis. Am Fam Physician. 2011;84(9):1027-1033.

14. Lindbloom BJ, James ER, McGarvey WC. Osteomyelitis of the foot and ankle: diagnosis, epidemiology, and treatment. Foot Ankle Clin. 2014; 19(3):569-588.

15. Panteli M, Puttaswamaiah R, Lowenberg DW, Giannoudis PV. Malignant transformation in chronic osteomyelitis: recognition and principles of management. J Am Acad Orthop Surg. 2014;22(9):586-594.

16. Senneville E, Nguyen S. Current pharmacotherapy options for osteomyelitis: convergences, divergences and lessons to be drawn. Expert Opin Pharmacother. 2013;14(6):723-734.

17. Jacqueline C, Caillon J. Impact of bacterial biofilm on the treatment of prosthetic joint infections. J Antimicrob Chemother. 2014;69(Suppl 1): i37-i40.

18. Valour F, Bouaziz A, Karsenty J, et al; Lyon BJI Study Group. Determinants of methicillin-susceptible Staphylococcus aureus native bone and joint infection treatment failure: a retrospective cohort study. BMC Infect Dis. 2014;14(1):443.

19. Fleiter $\mathrm{N}$, Walter $\mathrm{G}, \mathrm{Bösebeck} \mathrm{H}$, et al. Clinical use and safety of a novel gentamicin-releasing resorbable bone graft substitute in the treatment of osteomyelitis/osteitis. Bone Joint Res. 2014;3(7):223-229.

20. Rodvold KA, McConeghy KW. Methicillin-resistant Staphylococcus aureus therapy: past, present, and future. Clin Infect Dis. 2014; 58(Suppl 1):S20-S27.

21. Rostas SE, Kubiak DW, Calderwood MS. High-dose intravenous vancomycin therapy and the risk of nephrotoxicity. Clin Ther. 2014;36(7): 1098-1101.

22. Tan HL, Lin WT, Tang TT. The use of antimicrobial-impregnated PMMA to manage periprosthetic infections: controversial issues and the latest developments. Int J Artif Organs. 2012;35(10):832-839.

23. Babu RJ, Sathigari S, Kumar MT, Pandit JK. Formulation of controlled release gellan gum macro beads of amoxicillin. Curr Drug Deliv. 2010; $7(1): 36-43$.

24. Cao Z, Jiang D, Yan L, Wu J. In vitro and in vivo osteogenic activity of the novel vancomycin-loaded bone-like hydroxyapatite/poly(amino acid) scaffold. J Biomater Appl. 2016;30(10):1566-1577.
International Journal of Nanomedicine

\section{Publish your work in this journal}

The International Journal of Nanomedicine is an international, peerreviewed journal focusing on the application of nanotechnology in diagnostics, therapeutics, and drug delivery systems throughout the biomedical field. This journal is indexed on PubMed Central, MedLine, CAS, SciSearch ${ }^{\circledR}$, Current Contents ${ } /$ Clinical Medicine,

\section{Dovepress}

Journal Citation Reports/Science Edition, EMBase, Scopus and the Elsevier Bibliographic databases. The manuscript management system is completely online and includes a very quick and fair peer-review system, which is all easy to use. Visit http://www.dovepress.com/ testimonials.php to read real quotes from published authors. 